\title{
The electronic whiteboard in the Combined Assessment Unit - innovation in flow, governance and process efficiency
}

\author{
Authors: Elaine Clark, Mahanth Manuel and Emma Ramsay
}

\begin{abstract}
Aims
This abstract outlines the development of the e-whiteboard to improve the process efficiency during delivery of patient care in the new Combined Assessment Unit, University Hospital Crosshouse near Kilmarnock in Scotland.
\end{abstract}

\section{Methods}

The Combined Assessment Unit (CAU) opened in April 2016. It aims to deliver high-quality, evidence-based care to patients in a person-centred, safe and effective manner by early assessment, senior clinical-decision making and treatment.

The CAU is fully committed to delivering the three quality standards (safe, effective and person-centred care) as set out in the quality strategy. However, over the next few years the demands for health and social care and how they will be delivered, will be radically different. The need for process efficiency has never been greater.

\section{Objectives}

> Provide reference point for staff in CAU to access information and planned location status of patients in the department and expected to arrive.

> Patients to be prioritised for treatment pre-arrival by indicating red flag symptoms or clinical concerns from telephone referrals.

> Patients to be prioritised by displaying National Early Warning Scoring system (NEWS) and arrival times.

> Doctor / advanced nurse practitioner (ANP) to visualise electronically which patients are clerked by their colleagues.

> At-a-glance information of who/how many needs clerking in and consultant review.

$>$ ED to have a real time view of CAU capacity status and plan patient transfer arrangements.

> Stream patients to the right area in CAU after triage in initial assessment area.

> Identify frail patients requiring allied health professional and social care partnership early intervention.

$>$ ED Radiology department identify and prioritise imaging requests.

Using these objectives we designed a system following the complete patient journey capturing real world data the current Scottish Patient

Author: NHS Ayrshire \& Arran University Hospital Crosshouse, UK
Management System could not. This data includes time of referral to department, mode of transport, NEWS and red flag identification, time of arrival, bed allocation, time to review of a junior doctor or ANP, time to first consultant review, SPSP data such as medicines reconciliation and venous thromboembolism (VTE) prophylaxis, a task list for clinical support workers, direct link to radiology when $\mathrm{X}$-ray requested, direct link to pharmacy for discharge prescriptions, a frailty index score, an estimated date of discharge, and a bed allocation box for optimal bed management and allocation of specialty patients along with a free text general comments box. The system also allows pharmacy to pick up completed discharge letters to process the take-home medicines without involving phone calls. From the data we can extrapolate key performance indicators along with areas of clinical governance and performance.

The key steps taken to make this project a reality were multiple meetings with stakeholders to agree a process by projecting the benefits of process efficiency and time efficiency. Multiple demos of the electronic whiteboard (eWB) at development stage and introducing eWB in junior doctors' induction contributed to the success of this innovation.

\section{Results}

The results from business intelligence now show us key performance areas such as a reduction in length of stay, reduced time to be clerked in and seen by a consultant, reduced time waiting for a porter and an X-ray, and a reduction in waiting for pharmacy.

\section{Conclusions}

The opinion of the authors is that the eWB has undoubtedly given us key performance indicators in terms of time to review, consultation, discharge rate and Length of stay in department.

It also has allowed us to achieve our objectives in recording of governance issues such as medicines reconciliation, VTE prophylaxis and identification of sick patients along with the improved flow management and streamlining of timely investigations such as bloods, X-ray, and electrocardiography. It has allowed us to identify workforce requirements and training needs of staff. It has improved relationships and flow management with key stakeholders in the emergency department, X-ray, social work, physiotherapy, occupational therapy, and pharmacy departments. 